KYUSHU-HET-4

SAGA-HE-42

\title{
Chiral Invariance and Species Doublers in Generic Fermion Models on the Lattice
}

\author{
Koichi Funakubo* and Taro Kashiwa \\ *Department of Physics, Saga University, Saga, 840 JAPAN \\ Department of Physics, Kyushu University, Fukuoka, 812 JAPAN
}

\begin{abstract}
Discussions are made on the structures of chirally invariant lattice actions without any restriction of hermiticity. With the help of the Ward-Takahashi identity a general conclusion can be derived that there must be species doublers in any chirally invariant model provided that the model is chosen as well-regularized, that is, there is no singularity in the propagator after introducing fermion mass on the lattice. Various examples are discussed to pick up better models defined in the sense that the number of species doubler is smaller than that of the naive Dirac action.
\end{abstract}

$4 / 92$ 


\section{Introduction}

Since the advent of the no-go theorem of Nielsen and Ninomiya [1], people has struggled to put handed fermions on the lattice[2]. If this would be done, the nonperturbative treatment becomes possible to obtain the top quark mass and the baryon number generation in the standard model and to reduce some problems in the technicolor models. The theorem tells us that any chirally symmetric action with (i) locality (ii) translational invariance and (iii) hermiticity must always have equal number of left and right handed fermions (species doublers). In other words, we cannot help breaking a chiral invariance if we throw away those unwanted particles: Wilson [3] introduced the so-called Wilson term which breaks the chiral symmetry. The situation is the same in the case of Majoranatype fermion [4]. So far attempts have been made to lift the conditions (i) and/or (ii): the introduction of (Higgs) scalars [5] [6] [7] to the fermion action, yielding chiral gauge models, can be regarded as a non-local action after being integrated out with respect to those scalars. (Also there exists free (right) handed fermion in these approachs $[8]$.) The adoption of a random lattice can be classified in a similar class since in this approach the random variable behaves as a scalar field [9]. The mirror fermion method [10], uses an additional (mirror) fermion, which leads us again a non-local action after the integration of the mirror fermion(again with free redundant fermions).

Contrary to the above, there have been very few serious efforts [11] for lifting the condition (iii); hermiticity. The reason is that it is hard to deal with functions of complex variables if we abandon the hermiticity. Indeed it is very difficult to prove the no-go theorem in nonhermitian cases. However it would be economical in the sense that there is no need for the introduction of additional degrees of freedom to get a chirally symmetric model by throwing away the hermiticity. In this paper, we study the structure of chirally invariant lattice action with the help of Ward-Takahashi identity [12]. So far the existence of species doublers in a chirally symmetric and nonhermitian model has been anticipated by ref. [11] but we need a more general argument. Our strategy is as follows:

Step 1: Knowing that; propagator behaves $\sum_{\mu} i \gamma_{\mu} p_{\mu}+M$ around $p=0$ whose contribution to the Ward-Takahashi identity gives a well-known anomaly [13]

Step 2: Knowing that; on the lattice any chirally symmetric model does not have anomaly.

Step 3: Thus there must be the other zero of the inverse propagator which cancels the anomaly from $p=0$. (This is species doubler.) 
This leads us to the conclusion that if a model is well-regularized there should be species doublers in any chirally invariant model, which generalizes the no-go theorem to include nonhermitian actions.

In section 2, we set up a general form of chirally invariant actions and list some examples. In section 3, the Ward-Takahashi identity and the way to the continuum limit are discussed. Detailed calculations in two dimensions are then performed for general models with chiral symmetry in section 4 to illustrate our conclusion. The final section is devoted to further discussions. In the appendix, we present a brief introduction of the reflection positivity which is necessary to define the hermiticity on the lattice.

\section{General Form of Fermion Actions}

We write a general fermion action in $d$ dimensions as

$$
\begin{aligned}
I= & -\sum_{n} \sum_{\mu=1}^{d}\left\{\sum _ { k \geq 1 } \left[\bar{\psi}(n) \Gamma_{\mu}^{(+; k)} U_{\mu}(n) U_{\mu}(n+\mu) \cdots U_{\mu}(n+(k-1) \mu) \psi(n+k \mu)\right.\right. \\
& \left.\left.+\bar{\psi}(n+k \mu) \Gamma_{\mu}^{(-; k)} U_{\mu}^{\dagger}(n+(k-1) \mu) U_{\mu}^{\dagger}(n+(k-2) \mu) \cdots U_{\mu}^{\dagger}(n) \psi(n)\right]\right\} \\
& -\sum_{n} \bar{\psi}(n)\left(\Gamma^{(0)}+M\right) \psi(n),
\end{aligned}
$$

where $\Gamma_{\mu}^{( \pm ; k)}$ and $\Gamma^{(0)}$ are made from $\gamma$-matrices and $k$ is an integer running within some finite range to satisfy locality. (A more general case may be considered; where $\bar{\psi}(n)$ and $\psi(n+\cdots)$ are not located on a straight line such as $\bar{\psi}(n) \cdots \psi\left(n+k_{1} \mu+k_{2} \nu\right)(\mu \neq \nu)$. We shall not adopt such a model, since the choice of the link variables to connect them is not unique.) $U_{\mu}(n)$ is the usual link variable

$$
U_{\mu}(n)=\mathrm{e}^{i A_{\mu}(n)},
$$

where the coupling constant has been absorbed in the definition of gauge fields $A_{\mu}$. We take all quantities dimensionless such that

$$
\begin{aligned}
\psi(n) & \equiv a^{(d-1) / 2} \tilde{\psi}(x), \\
A_{\mu}(n) & \equiv a \tilde{A}_{\mu}(x),
\end{aligned}
$$

where $a$ is the lattice spacing, $x_{\mu}=a n_{\mu}$, and a tilde denotes the dimensional continuum quantity. In the same manner, the dimensionless mass(-matrix) is given by

$$
M=a \tilde{m},
$$


which plays the role of an infrared regulator: the infrared divergence is the only remaining singularity on the lattice. If the action is chosen properly (this must be checked since we lift the hermiticity in the following) we can study the continuum behavior of Feynman integrals by taking $M \rightarrow 0$, that is, $a \rightarrow 0$ with $\tilde{m}$ being fixed.

For later convenience, we now calculate the Fourier transformations of the propagator and the vertices. To this end, let us write the action (2.1) as

$$
I=-\sum_{m, n} \bar{\psi}(m) S^{-1}(m, n) \psi(n)
$$

with

$$
\begin{aligned}
S^{-1}(m, n) \equiv & \sum_{\mu, k \geq 1}\left[\delta_{m+k \mu, n} \Gamma_{\mu}^{(+; k)} U_{\mu}(m) \cdots U_{\mu}(m+(k-1) \mu)\right. \\
& \left.+\delta_{m, n+k \mu} \Gamma_{\mu}^{(-; k)} U_{\mu}^{\dagger}(n+(k-1) \mu) \cdots U_{\mu}^{\dagger}(n)\right] \\
& +\delta_{m, n}\left(\Gamma^{(0)}+M\right) .
\end{aligned}
$$

We then decompose $S^{-1}(m, n)$ into

$$
S^{-1}(m, n)=S_{0}^{-1}(m, n)+\Sigma(m, n)
$$

where

$$
\left.S_{0}^{-1}(m, n) \equiv S^{-1}(m, n)\right|_{A_{\mu}=0}
$$

thus

$$
\begin{aligned}
\Sigma(m, n) \equiv & \sum_{\mu, k \geq 1}\left\{\delta_{m+k \mu, n} \Gamma_{\mu}^{(+; k)}\left[U_{\mu}(m) \cdots U_{\mu}(m+(k-1) \mu)-1\right]\right. \\
& \left.+\delta_{m, n+k \mu} \Gamma_{\mu}^{(-; k)}\left[U_{\mu}^{\dagger}(n+(k-1) \mu) \cdots U_{\mu}^{\dagger}(n)-1\right]\right\} .
\end{aligned}
$$

$S_{0}^{-1}(m, n)$ and $\Sigma(m, n)$ are called the inverse propagator and the vertex respectively. Using

$$
\psi(n)=\int_{p} \mathrm{e}^{i p n} \psi(p)
$$

with

$$
p n \equiv \sum_{\mu} p_{\mu} n_{\mu}, \quad \int_{p} \equiv \int_{-\pi}^{\pi} \frac{d^{d} p}{(2 \pi)^{d}}
$$

we get the momentum representation of (2.8);

$$
S_{0}^{-1}(p)=\sum_{\mu, k \geq 1}\left(\Gamma_{\mu}^{(+; k)} \mathrm{e}^{i k p_{\mu}}+\Gamma_{\mu}^{(-; k)} \mathrm{e}^{-i k p_{\mu}}\right)+\Gamma^{(0)}+M
$$


Define a divisor $\mathcal{D}_{0}(p)$ such that

$$
S_{0}^{-1}(p) \mathcal{D}_{0}(p)=\Delta^{-1}(p) \mathbf{1}
$$

where $\Delta^{-1}(p)$ is the scalar inverse propagator. Then

$$
S_{0}(p)=\Delta(p) \mathcal{D}_{0}(p),
$$

which tells us that any singularity of the propagator $S_{0}(p)$ is controlled by $\Delta(p)$.

The Fourier transformation of the vertex can be obtained by use of the Taylor expansion with respect to $A_{\mu}$ as

$$
\begin{aligned}
& \sum_{m, n} \mathrm{e}^{-i q m} \sum(m, n) \mathrm{e}^{i p n} \\
= & \sum_{N=1}^{\infty} \frac{1}{N !}\left\{\sum_{k \geq 1}\left[\sum_{n} \mathrm{e}^{i(p-q)(n+k \mu / 2)}\left(\bar{A}_{\mu}^{(k)}(n)\right)^{N}\right] v_{\mu}^{(k ; N)}\left(\frac{p+q}{2}\right)\right\},
\end{aligned}
$$

where

$$
\bar{A}_{\mu}^{(k)}(n) \equiv \frac{1}{k} \sum_{J=0}^{k-1} A_{\mu}(n+J \mu),
$$

and

$$
v_{\mu}^{(k ; N)}(p) \equiv \frac{\partial^{N}}{\partial p_{\mu}^{N}}\left[\Gamma_{\mu}^{(+; k)} \mathrm{e}^{i k p_{\mu}}+\Gamma_{\mu}^{(-; k)} \mathrm{e}^{-i k p_{\mu}}\right] .
$$

Inspecting (2.16) and (2.11), we can recognize the relationship between the vertex and the inverse propagator. Furthermore $A_{\mu}$ is supposed to be a smooth function under $a \rightarrow 0$;

$$
A_{\mu}(n+J \mu) \simeq A_{\mu}(n)+O(a), \quad(a \rightarrow 0)
$$

to yield instead of (2.14),

$$
\begin{aligned}
& \sum_{m, n} \mathrm{e}^{-i q m} \Sigma(m, n) \mathrm{e}^{i p n} \\
= & \sum_{N=1}^{\infty} \frac{1}{N !} \sum_{n}\left(A_{\mu}(n)\right)^{N} \mathrm{e}^{i(p-q) n} v_{\mu}^{(N)}\left(\frac{p+q}{2}\right)+O(a),
\end{aligned}
$$

where

$$
v_{\mu}^{(N)}(p) \equiv \frac{\partial^{N}}{\partial p_{\mu}^{N}} S_{0}^{-1}(p),
$$

since the $k$ 's sum in (2.14) and (2.15) can be performed. (2.19) is the lattice Ward relation. 
Now we study various situations for the general fermion action (2.1):

(I) Chiral Invariance (when $M=0$ ):

$$
\left.\left\{\gamma_{5}, \Gamma_{\mu}^{( \pm ; k)}\right\}=\left\{\gamma_{5}, \Gamma^{(0)}\right\}=0 \quad \text { (for all } k \text { and } \mu\right)
$$

In this case the inverse propagator is given by in $d=2$

$$
\begin{aligned}
& S_{0}^{-1}(p)=i \gamma \cdot F(p)+M \\
& \Delta^{-1}(p)=F^{2}+M^{2}
\end{aligned}
$$

and in $d=4$

$$
\begin{aligned}
& S_{0}^{-1}(p)=i \gamma \cdot F(p)+\gamma \gamma_{5} \cdot G(p)+M \\
& \Delta^{-1}(p)=\left(F^{2}-G^{2}+M^{2}\right)^{2}+4 M^{2} G^{2}+4(F \cdot G)^{2}
\end{aligned}
$$

where use has been made of the abbreviation;

$$
\begin{aligned}
F^{2} & \equiv \sum_{\mu} F_{\mu}{ }^{2}, & G^{2} & \equiv \sum_{\mu} G_{\mu}{ }^{2} \\
\gamma \cdot F(p) & \equiv \sum_{\mu} \gamma_{\mu} F_{\mu}(p), & \gamma \gamma_{5} \cdot G(p) & \equiv \sum_{\mu} \gamma_{\mu} \gamma_{5} G_{\mu}(p) .
\end{aligned}
$$

(II) Hermiticity: the reflection positivity tells us that at least the following conditions should be fulfilled simultaneously:

$$
\begin{aligned}
& \gamma_{d} \Gamma_{i}^{( \pm ; k)^{\dagger}} \gamma_{d}=\Gamma_{i}^{(\mp ; k)} \quad \text { for } i=1,2, \cdots, d-1 \\
& \gamma_{d} \Gamma_{d}^{( \pm ; k)^{\dagger}} \gamma_{d}=\Gamma_{d}^{( \pm ; k)}, \quad \gamma_{d} \Gamma^{(0)^{\dagger}} \gamma_{d}=\Gamma^{(0)} .
\end{aligned}
$$

as well as $k \leq 1$.

We now impose some conditions to our general action.

(a) Naive Continuum Limit: the inverse propagator (2.11), (2.21), (2.22) behaves such that

$$
S_{0}^{-1}(p) \underset{p \rightarrow 0}{\rightarrow} i \gamma \cdot p+M
$$

(b) Regularization Free: there should be no singularity in $S_{0}(p)$ as long as $M \neq 0$. The condition reads from (2.13)

$$
\left|\Delta^{-1}(p)\right| \supsetneqq 0 \quad \text { for } M^{2} \supsetneqq 0 .
$$

1 Additional conditions are also necessary but the form of them is not so simple; thus we relegate it to the appendix. 
In a chirally invariant case, this turns out, according to (2.21) and (2.22), to be

$$
\left|F^{2}(p)+M^{2}\right| \supsetneqq 0, \quad \text { for } d=2,
$$

or

$$
\left|\left(F^{2}-G^{2}+M^{2}\right)^{2}+4 M^{2} G^{2}+4(F \cdot G)^{2}\right| \supsetneqq 0, \quad \text { for } d=4 \text {. }
$$

(c) Pole Singularity: when $M=0$ any singularity of the propagator $S_{0}(p)$, that is, of $\Delta(p)$, must be a pole.

(d) Direction Interchange Symmetry(DIS): the form of $\Gamma_{\mu}^{( \pm ; k)}$ is unchanged after an interchange of $\mu$-direction.

Here we explain these conditions: the condition (a) is a fundamental requirement for any lattice model. (b) is especially necessary in nonhermitian cases since $S_{0}^{-1}(p)$ may contain complex numbers in general. Unless this is satisfied, we need an additional regularization even on the lattice. Furthermore it is necessary for the condition (c) to be fulfilled since otherwise there need additional (and maybe very cumbersome) methods to estimate the lattice Feynman integral. As for the condition (d); this is a statement of the relativistic invariance on the lattice. Without this we have less symmetric model to recover the continuum limit much slower. (Note that this condition is different from the lattice rotation symmetry. See below.)

Now we check some explicit examples:

(1) Naive Dirac Case: we take

$$
\Gamma_{\mu}^{(+; 1)}=\frac{\gamma_{\mu}}{2}, \quad \Gamma_{\mu}^{(-; 1)}=-\frac{\gamma_{\mu}}{2}, \quad \text { others }=0
$$

The action is

$$
\begin{aligned}
I^{(1)}= & -\sum_{n, \mu}\left\{\frac{1}{2}\left[\bar{\psi}(n) \gamma_{\mu} U_{\mu}(n) \psi(n+\mu)-\bar{\psi}(n+\mu) \gamma_{\mu} U_{\mu}^{\dagger}(n) \psi(n)\right]\right\} \\
& -\sum_{n} \bar{\psi}(n) M \psi(n),
\end{aligned}
$$

which is, from (I) and (II), chirally invariant and hermitian. The inverse propagator is given by

$$
\begin{gathered}
S_{0}^{-1}(p)=i \gamma \cdot \sin p+M \\
\Delta^{-1}(p)=(\sin p)^{2}+M^{2}
\end{gathered}
$$


with $(\sin p)^{2} \equiv \sum_{\mu} \sin ^{2} p_{\mu} . \Delta^{-1}(p)$ has $2^{D}$ zeros at $p_{\mu}=0$ or $\pi$, around which

$$
\Delta^{-1}(p) \underset{p_{\mu} \rightarrow 0, \pi}{\longrightarrow} p^{2}+M^{2}
$$

All the conditions $(\mathrm{a}) \sim(\mathrm{d})$ are apparently satisfied.

(2) Wilson Case: we take

$$
\begin{aligned}
& \Gamma^{(0)}=r d, \quad \Gamma_{\mu}^{(+; 1)}=\frac{\gamma_{\mu}-r}{2}, \\
& \Gamma_{\mu}^{(-; 1)}=-\frac{\gamma_{\mu}+r}{2}, \quad \text { others }=0, \quad \mathrm{r} \in \mathbf{R} .
\end{aligned}
$$

The action is

$$
\begin{aligned}
I^{(2)}= & -\sum_{n, \mu}\left\{\frac{1}{2}\left[\bar{\psi}(n) \gamma_{\mu} U_{\mu}(n) \psi(n+\mu)-\bar{\psi}(n+\mu) \gamma_{\mu} U_{\mu}^{\dagger}(n) \psi(n)\right]\right. \\
& \left.-\frac{r}{2}\left[\bar{\psi}(n) U_{\mu}(n) \psi(n+\mu)+\bar{\psi}(n+\mu) U_{\mu}^{\dagger}(n) \psi(n)-2 \bar{\psi}(n) \psi(n)\right]\right\} \\
& -\sum_{n} \bar{\psi}(n) M \psi(n) .
\end{aligned}
$$

This model is not chirally invariant as long as $r \neq 0$. (When $r \rightarrow 0$, this becomes to the case (1).) If $0 \leq r^{2} \leq 1$, according to the reflection positivity 2 , hermiticity is satisfied. The inverse propagator is

$$
\begin{gathered}
S_{0}^{-1}(p)=i \gamma \cdot \sin p+r C(p)+M \\
\Delta^{-1}(p)=(\sin p)^{2}+(M+r C(p))^{2} \\
C(p) \equiv \sum_{\mu}\left(1-\cos p_{\mu}\right) .
\end{gathered}
$$

Due to the $C(p)$ term, $\Delta^{-1}(p)$ has only one zero at $p^{(0)}=(0,0, \ldots)$ where

$$
\Delta^{-1}(p) \underset{p \rightarrow p^{(0)}}{\longrightarrow} p^{2}+M^{2}
$$

(3) Alonso-Boucaudo-Cortes-Rivas(ABCR) Model[14]: we take

$$
\begin{gathered}
\Gamma_{\mu}^{(+; 1)}=-\frac{1+i}{2} \gamma_{\mu}+\frac{i}{2 d} \sum_{\nu} \gamma_{\nu} \\
\Gamma_{\mu}^{(-; 1)}=\frac{1-i}{2} \gamma_{\mu}+\frac{i}{2 d} \sum_{\nu} \gamma_{\nu}, \quad \text { others }=0 .
\end{gathered}
$$

2 See the appendix again. 
The action is

$$
\begin{aligned}
I^{(3)}= & -\sum_{n, \mu}\left\{\frac { 1 } { 2 } \left[\bar{\psi}(n)\left\{(1+i) \gamma_{\mu}-\frac{i}{d} \sum_{\nu} \gamma_{\nu}\right\} U_{\mu}(n) \psi(n+\mu)\right.\right. \\
& \left.-\bar{\psi}(n+\mu)\left\{(1-i) \gamma_{\mu}+\frac{i}{d} \sum_{\nu} \gamma_{\nu}\right\} U_{\mu}^{\dagger}(n) \psi(n)\right] \\
& -\sum_{n} \bar{\psi}(n) M \psi(n),
\end{aligned}
$$

which is hermitian as well as chirally invariant. The inverse propagator is

$$
\begin{gathered}
S_{0}^{-1}(p)=i \gamma \cdot F(p)+M, \quad \Delta^{-1}(p)=F^{2}+M^{2}, \\
F_{\mu}(p) \equiv \sin p_{\mu}+1-\cos p_{\mu}-\frac{2}{d} C(p),
\end{gathered}
$$

whose zeros are

$$
p^{(0)}=(0,0, \ldots), \quad p^{(1)}=(\pi / 2, \pi / 2, \ldots) .
$$

Around these

$$
\Delta^{-1}(p) \underset{p \rightarrow p^{(0)}, p^{(1)}}{\longrightarrow} p^{2}+M^{2} .
$$

Thus this model satisfies all the conditions $(\mathrm{a}) \sim(\mathrm{d})$. As previously stated, this model does not have a lattice rotation symmetry [14 but does the Direction Interchange Symmetry(DIS).

(4) Nonhermitian Case (i)3:

$$
\begin{aligned}
& \Gamma^{(0)}=\sum_{\mu} \gamma_{\mu} \gamma_{5}, \quad \Gamma_{\mu}^{(+; 1)}=\frac{\gamma_{\mu}\left(1-\gamma_{5}\right)}{2}, \\
& \Gamma_{\mu}^{(-; 1)}=-\frac{\gamma_{\mu}\left(1+\gamma_{5}\right)}{2}, \quad \text { others }=0 .
\end{aligned}
$$

The action is

$$
\begin{aligned}
I^{(4)}=-\sum_{n, \mu}\left\{\frac{1}{2}\left[\bar{\psi}(n) \gamma_{\mu} U_{\mu}(n) \psi(n+\mu)-\bar{\psi}(n+\mu) \gamma_{\mu} U_{\mu}^{\dagger}(n) \psi(n)\right]\right. \\
\quad-\frac{1}{2}\left[\bar{\psi}(n) \gamma_{\mu} \gamma_{5} U_{\mu}(n) \psi(n+\mu)+\bar{\psi}(n+\mu) \gamma_{\mu} \gamma_{5} U_{\mu}^{\dagger}(n) \psi(n)\right. \\
\left.\left.\quad-2 \bar{\psi}(n) \gamma_{\mu} \gamma_{5} \psi(n)\right]\right\} r-\sum_{n} \bar{\psi}(n) M \psi(n),
\end{aligned}
$$

3 The cases (4) and (5) are not only simple but also obtainable from the operator formalism by using fermion coherent states as is the Wilson cases 15$]$. 
which is chirally invariant but nonhermitian. The inverse propagator is given by in $d=2$

$$
\begin{aligned}
& S_{0}^{-1}(p)=\sum_{\mu, \nu} i \gamma_{\mu}\left[\sin p_{\mu}+\epsilon_{\mu \nu}\left(1-\cos p_{\nu}\right)\right]+M \\
& \Delta^{-1}(p)=\sum_{\mu}\left[\sin p_{\mu}+\sum_{\nu} \epsilon_{\mu \nu}\left(1-\cos p_{\nu}\right)\right]^{2}+M^{2} \\
& =2\left(1-\cos p_{1}\right)\left(1-\sin p_{2}\right)+2\left(1-\cos p_{2}\right)\left(1+\sin p_{1}\right)+M^{2},
\end{aligned}
$$

whose zeros (when $M=0$ ) are

$$
p^{(0)}=(0,0), \quad p^{(1)}=(-\pi / 2, \pi / 2) .
$$

Around these

$$
\Delta^{-1}(p) \underset{p \rightarrow p^{(0)}, p^{(1)}}{\rightarrow} p^{2}+M^{2}
$$

Thus all the conditions are satisfied. In the four dimensional case

$$
\begin{aligned}
& S_{0}^{-1}(p)=\sum_{\mu}\left[i \gamma_{\mu} \sin p_{\mu}+\gamma_{\mu} \gamma_{5}\left(1-\cos p_{\mu}\right)\right]+M \\
& \Delta^{-1}(p)=\left(F^{2}-G^{2}+M^{2}\right)^{2}+4 M^{2} G^{2}+4(F \cdot G)^{2}
\end{aligned}
$$

with $F_{\mu}=\sin p_{\mu}$ and $G_{\mu}=1-\cos p_{\mu}$. When $M=0$, the zeros should satisfy

$$
\begin{aligned}
& \sum_{\mu} \cos p_{\mu}\left(1-\cos p_{\mu}\right)=0 \\
& \sum_{\mu} \sin p_{\mu}\left(1-\cos p_{\mu}\right)=0 .
\end{aligned}
$$

Introducing $x$ and $y$ such that

$$
p=(x,-x, y,-y) \text { or }(x, y,-x,-y) \text { or }(x, y,-y,-x) \text {, }
$$

we can see the second equation is trivially satisfied and the first one becomes

$$
\cos x(1-\cos x)+\cos y(1-\cos y)=0
$$

to give a circle in $(\cos x, \cos y)$-plane. Thus the zeros of (2.46) are described by the semicircle defined by the intersection of (2.49) and the domain $\{|\cos x| \leq$ $1\} \cap\{|\cos y| \leq 1\}$. (See Fig.1.) As can be read from the figure, $p^{(0)}=(0,0,0,0)$ 
is isolated but additional zeros are not poles but rather a cut which breaks the condition (c).

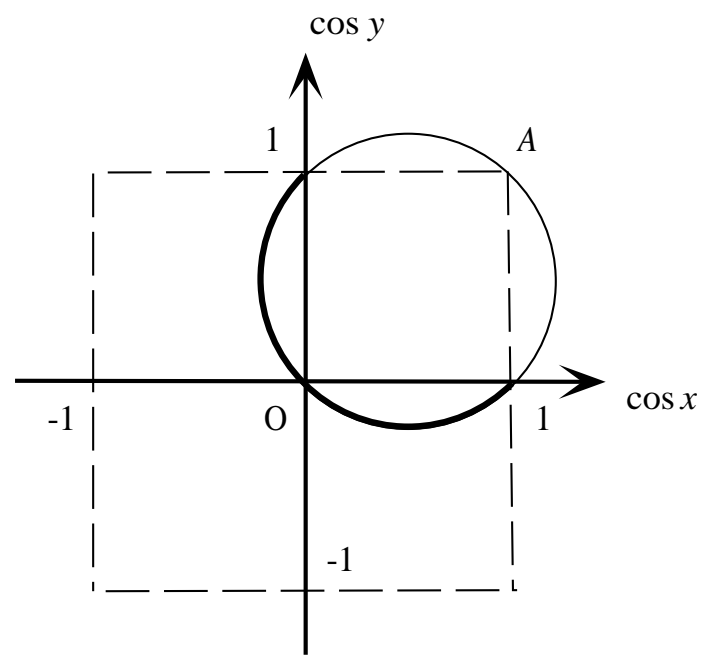

Fig.1: Allowed region of (2.49)

Point A corresponds to $p^{(0)}=(0,0,0,0$,$) .$

(5) Nonhermitian Case (ii): we consider

$$
\Gamma^{(0)}=-\sum_{\mu} \gamma_{\mu}, \quad \Gamma_{\mu}^{(+; 1)}=\gamma_{\mu}, \quad \text { others }=0
$$

to give

$$
I^{(5)}=-\sum_{n, \mu} \bar{\psi}(n) \gamma_{\mu}\left(U_{\mu}(n) \psi(n+\mu)-\psi(n)\right)-\sum_{n} \bar{\psi}(n) M \psi(n)
$$

This is also chirally invariant and nonhermitian. The inverse propagator in this case is given by

$$
S_{0}^{-1}(p)=\sum_{\mu} \gamma_{\mu}\left(\mathrm{e}^{i p_{\mu}}-1\right)+M, \quad \Delta^{-1}(p)=\sum_{\mu}\left(\mathrm{e}^{i p_{\mu}}-1\right)^{2}+M^{2}
$$

Then

$$
\begin{aligned}
& \left|\Delta^{-1}(p)\right| \\
& =\sqrt{\left[\sum_{\mu} 2 \cos p_{\mu}\left(1-\cos p_{\mu}\right)-M^{2}\right]^{2}+4\left[\sum_{\mu} \sin p_{\mu}\left(1-\cos p_{\mu}\right)\right]^{2}},
\end{aligned}
$$

which vanishes at $p=\left(p_{0},-p_{0}\right)$ with $p_{0}$ being given by $\cos p_{0}=\left(1 \pm \sqrt{1-M^{2}}\right) / 2$ in $d=2$ for example. Therefore this model has a singularity even when $M \neq 0$. 


\section{Chiral Ward-Takahashi Identity and Continuum Limit}

In this section, we discuss the chiral Ward-Takahashi identity and its behavior in the continuum limit, $a \rightarrow 0$.

The chiral transformation,

$$
\psi(n) \mapsto \mathrm{e}^{i \theta(n) \gamma_{5}} \psi(n), \quad \bar{\psi}(n) \mapsto \bar{\psi}(n) \mathrm{e}^{i \theta(n) \gamma_{5}}
$$

applying to integration variables of the partition function,

$$
Z[A] \equiv \int \prod_{n}[d \psi(n) d \bar{\psi}(n)] \exp [I[\psi, \bar{\psi} ; A]]
$$

with $I$ being given by (2.1) leads us to the Ward-Takahashi identity,

$$
\left\langle\left[\bar{\psi}(n) i \gamma_{5} \frac{\partial}{\partial \bar{\psi}(n)}+i \gamma_{5} \psi(n) \frac{\partial}{\partial \psi(n)}\right] I\right\rangle \equiv 0
$$

where

$$
\langle\mathcal{O}\rangle \equiv \int \prod_{n}[d \psi(n) d \bar{\psi}(n)] \mathcal{O} \mathrm{e}^{I} / Z[A] .
$$

Equation (3.3) can be read, by writing

$$
\begin{aligned}
J^{(+)}(n ; k) & \equiv \sum_{\mu} \bar{\psi}(n) i \gamma_{5} \Gamma_{\mu}^{(+; k)} U_{\mu}(n) \cdots U_{\mu}(n+(k-1) \mu) \psi(n+k \mu), \\
J^{(-)}(n ; k) & \equiv \sum_{\mu} \bar{\psi}(n+k \mu) i \gamma_{5} \Gamma_{\mu}^{(-; k)} U_{\mu}^{\dagger}(n+(k-1) \mu) \cdots U_{\mu}^{\dagger}(n) \psi(n),
\end{aligned}
$$

as

$$
\begin{aligned}
& \left\langle\sum_{\substack{k \geq 1 \\
\text { invariant }}}\left[\left(J^{(+)}(n ; k)-J^{(+)}(n-k ; k)\right)-\left(J^{(-)}(n ; k)-J^{(-)}(n-k ; k)\right)\right]\right\rangle \\
& \quad+\langle X(n)\rangle=-\left\langle\bar{\psi}(n) 2 i M \gamma_{5} \psi(n)\right\rangle,
\end{aligned}
$$

where

$$
\begin{aligned}
X(n) \equiv & \sum_{\substack{k \geq 1 \\
\text { noninvariant }}}\left[\left(J^{(+)}(n ; k)+J^{(+)}(n-k ; k)\right)\right. \\
& \left.+\left(J^{(-)}(n ; k)+J^{(-)}(n-k ; k)\right)\right]+\bar{\psi}(n)\left\{i \gamma_{5}, \Gamma^{(0)}\right\} \psi(n)
\end{aligned}
$$

Here we have divided the general action into chirally invariant and noninvariant parts characterized by $(2.20)$ and

$$
\left[\gamma_{5}, \Gamma_{\mu}^{( \pm ; k)}\right]=0, \quad \text { for noninvariant pieces. }
$$


Since $k$ 's sum is finite, the first term in the left-hand side of (3.6) vanishes when being summed up with respect to $n$ to yield

$$
\sum_{n}\langle X(n)\rangle=-\sum_{n}\left\langle\bar{\psi}(n) 2 i M \gamma_{5} \psi(n)\right\rangle .
$$

We require that this Ward-Takahashi identity must be fulfilled at every stage while taking continuum limit, $a \rightarrow 0$.

In the following, we study the right-hand side of (3.8) in terms of an $A_{\mu}$-expansion. To achieve this, we take the trace with respect to $\gamma$-matrices as well as the mass matrix (and also to gauge-group index, if any) then recall that $S(m, n)$ is given by (2.7) to find

$$
\begin{aligned}
-\sum_{n}\left\langle\bar{\psi}(n) 2 i M \gamma_{5} \psi(n)\right\rangle= & \sum_{n} \operatorname{Tr}\left[2 i M \gamma_{5} S(n, n)\right] \\
= & \sum_{n}\left[\mathcal{A}^{(0)}+\sum_{\mu} \int_{p} \mathrm{e}^{i p n} A_{\mu}(p) \mathcal{A}_{\mu}^{(1)}(p)\right. \\
& \left.+\frac{1}{2} \sum_{\mu, \nu} \int_{p, q} \mathrm{e}^{i(p+q) n} A_{\mu}(p) A_{\nu}(q) \mathcal{A}_{\mu \nu}^{(2)}(p, q)+O\left(A^{3}\right)\right]
\end{aligned}
$$

where

$$
A_{\mu}(p) \equiv \sum_{n} \mathrm{e}^{-i p n} A_{\mu}(n)
$$

and

$$
\begin{aligned}
\mathcal{A}^{(0)} \equiv & \int_{l} \operatorname{Tr}\left[2 i M \gamma_{5} S_{0}(l)\right], \\
\mathcal{A}_{\mu}^{(1)}(p) \equiv & -\int_{l} \operatorname{Tr}\left[2 i M \gamma_{5} S_{0}\left(l+\frac{p}{2}\right) v_{\mu}^{(1)}(l) S_{0}\left(l-\frac{p}{2}\right)\right], \\
\mathcal{A}_{\mu \nu}^{(2)}(p, q) \equiv & -\int_{l} \operatorname{Tr}\left[2 i M \gamma_{5} S_{0}\left(l+\frac{p+q}{2}\right) \delta_{\mu \nu} v_{\mu}^{(2)}(l) S_{0}\left(l-\frac{p+q}{2}\right)\right] \\
& +\int_{l} \operatorname{Tr}\left[2 i M \gamma_{5} S_{0}\left(l+\frac{p+q}{2}\right) v_{\mu}^{(1)}\left(l+\frac{q}{2}\right) S_{0}\left(l-\frac{p-q}{2}\right)\right. \\
& \left.\times v_{\nu}^{(1)}\left(l-\frac{p}{2}\right) S_{0}\left(l-\frac{p+q}{2}\right)\right] \\
& +\int_{l} \operatorname{Tr}\left[2 i M \gamma_{5} S_{0}\left(l+\frac{p+q}{2}\right) v_{\nu}^{(1)}\left(l+\frac{p}{2}\right) S_{0}\left(l+\frac{p-q}{2}\right)\right. \\
& \left.\times v_{\mu}^{(1)}\left(l-\frac{q}{2}\right) S_{0}\left(l-\frac{p+q}{2}\right)\right] .
\end{aligned}
$$

We calculate each term of the right-hand side of (3.9) under $a \rightarrow 0$. To this end we expand each coefficient around $p=0$. The power counting on the lattice [16] tells that the first 
derivative of $\mathcal{A}_{\mu}^{(1)}(p)$ is relevant in $d=2$ while the second derivative, $\partial^{2} \mathcal{A}_{\mu \nu}^{(2)}(p, q) / \partial p_{\alpha} \partial q_{\beta}$, is in $d=4$.

Let us calculate $\mathcal{A}_{\mu}^{(1)}(p)$ in the case of Wilson action (2.33) in two dimensions. Due to the chiral noninvariance, we have $X(n)$;

$$
\begin{gathered}
X(n)=-\frac{r}{2} \sum_{\mu}\left\{\left[\bar{\psi}(n) i \gamma_{5} U_{\mu}(n) \psi(n+\mu)+\bar{\psi}(n-\mu) i \gamma_{5} U_{\mu}(n-\mu) \psi(n)\right]\right. \\
+\left[\bar{\psi}(n+\mu) i \gamma_{5} U_{\mu}^{\dagger}(n) \psi(n)+\bar{\psi}(n) i \gamma_{5} U_{\mu}^{\dagger}(n-\mu) \psi(n-\mu)\right] \\
\left.-4 \bar{\psi}(n) i \gamma_{5} \psi(n)\right\} .
\end{gathered}
$$

Note that $\mathcal{A}^{(0)}=0$ because of the trace property. In view of $(2.34)$ we find

$$
\mathcal{A}_{\mu}^{(1)}(p)=-2 \int_{l} \Delta\left(l+\frac{p}{2}\right) \Delta\left(l-\frac{p}{2}\right)\left[4 M^{2} \sum_{\nu} \epsilon_{\mu \nu} \cos l_{\mu} \cos l_{\nu} \sin \frac{p_{\nu}}{2}+r N_{\mu}(l ; p)\right],
$$

where

$$
\begin{gathered}
N_{\mu}(l ; p) \equiv 2 M\left\{\sum_{\nu} \cos l_{\mu} \epsilon_{\mu \nu}\left[\sin \left(l+\frac{p}{2}\right)_{\nu} C\left(l-\frac{p}{2}\right)-\sin \left(l-\frac{p}{2}\right)_{\nu} C\left(l+\frac{p}{2}\right)\right]\right. \\
\left.+\sum_{\nu, \lambda} \sin l_{\mu} \epsilon_{\nu \lambda} \sin \left(l+\frac{p}{2}\right)_{\nu} \sin \left(l-\frac{p}{2}\right)_{\lambda}\right\} .
\end{gathered}
$$

We expand $\mathcal{A}_{\mu}^{(1)}(p)$ around $p=0$;

$$
\mathcal{A}_{\mu}^{(1)}(p)=\left.\frac{\partial \mathcal{A}_{\mu}{ }^{(1)}}{\partial p_{\nu}}\right|_{p=0} p_{\nu}+O\left(p^{3}\right),
$$

where

$$
\begin{aligned}
\left.\frac{\partial \mathcal{A}_{\mu}{ }^{(1)}}{\partial p_{\nu}}\right|_{p=0}= & -4 M^{2} \int_{l} \Delta(l)^{2}\left[\epsilon_{\mu \nu} \cos l_{\mu} \cos l_{\nu}\right] \\
& -4 M r \int_{l} \Delta(l)^{2}\left[\epsilon_{\mu \nu} \cos l_{\mu} \cos l_{\nu} C(l)\right. \\
& \left.-\sum_{\lambda}\left(\epsilon_{\mu \lambda} \sin l_{\nu} \cos l_{\mu}-\epsilon_{\nu \lambda} \sin l_{\mu} \cos l_{\nu}\right) \sin l_{\lambda}\right]
\end{aligned}
$$

In order to estimate the above integrals, we first recall that there is only one zero (2.35) then divide the integration region into $D_{\epsilon}\left(l^{(0)}\right)$ where

$$
D_{\epsilon}\left(l^{(0)}\right) \equiv\left\{l \mid\left(l-l^{(0)}\right)^{2} \leq \epsilon^{2}\right\}
$$


with $l^{(0)}=(0,0)$ and the rest, $D_{r}\left(l^{(0)}\right) \equiv[-\pi, \pi]^{2}-D_{\epsilon}\left(l^{(0)}\right)$. We find

$$
\lim _{M \rightarrow 0} M \int_{D_{r}\left(l^{(0)}\right)}[\cdots \cdots]=0
$$

since there is no singularity under the integration. Also

$$
\begin{gathered}
\lim _{M \rightarrow 0} M^{2} \int_{D_{\epsilon}\left(l^{(0)}\right)} \frac{d^{2} l}{(2 \pi)^{2}} \frac{1}{\left(l^{2}+M^{2}\right)^{2}}=\frac{1}{4 \pi} \\
\lim _{M \rightarrow 0} M \int_{D_{\epsilon}\left(l^{(0)}\right)} \frac{d^{2} l}{(2 \pi)^{2}} \frac{l^{2 m}}{\left(l^{2}+M^{2}\right)^{2}}=0, \quad \text { for } m \geq 1 .
\end{gathered}
$$

Using these, we obtain

$$
\begin{aligned}
\left.\lim _{M \rightarrow 0} \frac{\partial \mathcal{A}_{\mu}{ }^{(1)}}{\partial p_{\nu}}\right|_{p=0}= & \lim _{M \rightarrow 0}\left[-4 M^{2} \int_{D_{\epsilon}\left(l^{(0)}\right)} \frac{d^{2} l}{(2 \pi)^{2}} \frac{\epsilon_{\mu \nu}}{\left(l^{2}+M^{2}\right)^{2}}\right. \\
& \left.-4 M r \int_{D_{\epsilon}\left(l^{(0)}\right)} \frac{d^{2} l}{(2 \pi)^{2}} \frac{\epsilon_{\mu \nu} l^{2}-\sum_{\lambda}\left(\epsilon_{\mu \lambda} l_{\nu}-\epsilon_{\nu \lambda} l_{\mu}\right) l_{\lambda}}{\left(l^{2}+M^{2}\right)^{2}}\right] \\
= & -\frac{\epsilon_{\mu \nu}}{\pi} .
\end{aligned}
$$

From (3.20), the right hand side of (3.8) becomes, in view of (3.9) and (3.15), as

$$
\begin{aligned}
\lim _{a \rightarrow 0} \sum_{n}\langle X(n)\rangle & =\lim _{a \rightarrow 0} \sum_{n} \int_{p} \mathrm{e}^{i p n} A_{\mu}(p)\left(-\frac{1}{\pi} \epsilon_{\mu \nu} p_{\nu}\right) \\
& =i \int d^{2} x \frac{1}{\pi} \epsilon_{\mu \nu} \partial_{\nu} \tilde{A}_{\mu}(x),
\end{aligned}
$$

which reveals the correct anomaly relation in the continuum (by inserting (3.21) into (3.6));

$$
\partial_{\mu} J_{5 \mu}(x)=\frac{i}{\pi} \epsilon_{\mu \nu} \tilde{F}_{\mu \nu}-2 M J_{5}(x)
$$

with

$$
J_{5 \mu}(x) \equiv \tilde{\bar{\psi}}(x) i \gamma_{5} \gamma_{\mu} \tilde{\psi}(x), \quad J_{5}(x) \equiv \tilde{\bar{\psi}}(x) i \gamma_{5} \tilde{\psi}(x)
$$

Next let us consider what happens if $r=0$, that is, in the naive Dirac case: there is no $X(n)$ in the $\mathrm{W}$-T relation (3.8) to give

$$
\sum_{n}\left\langle\bar{\psi}(n) 2 i M \gamma_{5} \psi(n)\right\rangle=0
$$


$\mathcal{A}^{(0)}=0$ as the above. $\mathcal{A}_{\mu}^{(1)}(p)$ is found, by putting $r \rightarrow 0$ in $(\underline{3.13})$, as

$$
\mathcal{A}_{\mu}^{(1)}(p)=-4 M^{2} \sum_{\nu} \epsilon_{\mu \nu} p_{\nu} \int_{l} \Delta^{2}(l) \cos l_{\mu} \cos l_{\nu}+O\left(p^{3}\right) .
$$

Significance in this case is, as can be seen from (2.31), that there are four poles in $\Delta(l)$ when $M \rightarrow 0: l^{(1)}=(0, \pi), l^{(2)}=(\pi, 0)$ and $l^{(3)}=(\pi, \pi)$ other than $l^{(0)}=(0,0)$. Around these poles, the form of $\Delta(l)$ expressed by (2.31) is the same but $\cos l_{\mu}$ and $\cos l_{\nu} \operatorname{change}$ their sign to give

$$
\mathcal{A}_{\mu}^{(1)}(p)=-\frac{1}{\pi} \epsilon_{\mu \nu} p_{\nu}(1-1-1+1)=0 .
$$

Hence (3.22) is fulfilled. These additional poles are nothing but species doublers. Species doublers control the $W$-T identity in the (chirally invariant) naive Dirac case.

\section{General Case with Chiral Symmetry}

Let us discuss the general case with a chiral symmetry. In two dimensions, the most general chiral invariant propagator is given by (2.21);

$$
S_{0}^{-1}=i \gamma \cdot F(p)+M
$$

With the use of this, it is easily to see that

$$
\mathcal{A}^{(0)}=0
$$

because of the trace property. While $\mathcal{A}_{\mu}^{(1)}(p)$ is given

$$
\begin{aligned}
\mathcal{A}_{\mu}^{(1)}(p)= & -4 M^{2} \sum_{\alpha, \beta} \epsilon_{\alpha \beta} \int_{l} \Delta(l+p / 2) \Delta(l-p / 2) \\
& \times \frac{\partial F_{\alpha}(l)}{\partial l_{\mu}}\left[F_{\beta}(l+p / 2)-F_{\beta}(l-p / 2)\right],
\end{aligned}
$$

where

$$
\Delta(l) \equiv\left[F(l)^{2}+M^{2}\right]^{-1} .
$$

The Taylor expansion with respect to $p$ leads us to

$$
\mathcal{A}_{\mu}^{(1)}(p)=-4 M^{2} \sum_{\alpha, \beta, \nu} \epsilon_{\alpha \beta} p_{\nu} \int_{l} \Delta^{2}(l) \frac{\partial F_{\alpha}(l)}{\partial l_{\mu}} \frac{\partial F_{\beta}(l)}{\partial l_{\nu}}+O\left(p^{3}\right) .
$$


The propagator $\Delta(l)(4.3)$ has a pole at $l=l^{(0)} \equiv(0,0)$ when $M=0$ due to the condition (a) and (c) in section 2. Thus the contribution from the domain $D_{\epsilon}\left(l^{(0)}\right)$ is just the same as (3.18) and the condition (b) and (c) tells us that there might be another contribution from a pole, say $l^{(i)}$ :

$$
\begin{gathered}
\mathcal{A}_{\mu}^{(1)}(p)=-\lim _{M \rightarrow 0} 4 M^{2} \sum_{\alpha, \beta, \nu} \epsilon_{\alpha \beta} p_{\nu}\left[\int_{D_{\epsilon}\left(l^{(0)}\right)} \frac{\delta_{\alpha \mu} \delta_{\beta \nu}}{\left(l^{2}+M^{2}\right)^{2}}\right. \\
\left.+\sum_{i} \int_{D_{\epsilon}\left(l^{(i)}\right)} \Delta^{2}(l) \frac{\partial F_{\alpha}(l)}{\partial l_{\mu}} \frac{\partial F_{\beta}(l)}{\partial l_{\nu}}\right]+O\left(p^{3}\right) \\
=-\frac{1}{\pi} \epsilon_{\mu \nu} p_{\nu}-\lim _{M \rightarrow 0} 4 M^{2} \sum_{\alpha, \beta, \nu, i} \epsilon_{\alpha \beta} p_{\nu} \int_{D_{\epsilon}\left(l^{(i)}\right)} \Delta^{2}(l) \frac{\partial F_{\alpha}(l)}{\partial l_{\mu}} \frac{\partial F_{\beta}(l)}{\partial l_{\nu}}+O\left(p^{3}\right) .
\end{gathered}
$$

The Ward-Takahashi identity in this case is also given by (3.22) But if there would be no pole we would obtain

$$
\sum_{n}\left\langle\bar{\psi}(n) 2 i M \gamma_{5} \psi(n)\right\rangle \neq 0
$$

instead. Hence $\Delta(l)$ (4.3) must have additional pole(s) to cancel the first term of (4.5): there should be 'species doublers' even in nonhermitian cases 11 .

Let us study this situation in the explicit example (2.42), where

$$
F_{\mu}(p)=\sin p_{\mu}+\sum_{\nu} \epsilon_{\mu \nu}\left(1-\cos p_{\nu}\right)
$$

Thus in view of (2.44) and (2.45), we have two contributions from $l^{(0)}$ and $l^{(1)}$ to find that

$$
\begin{aligned}
\mathcal{A}_{\mu}^{(1)}(p)= & -4 M^{2} \sum_{\nu} \epsilon_{\mu \nu} p_{\nu} \int_{l} \Delta^{2}(l)\left(\cos l_{\mu} \cos l_{\nu}+\sin l_{\mu} \sin l_{\nu}\right) \\
= & -4 M^{2} \sum_{\nu} \epsilon_{\mu \nu} p_{\nu}\left[\int_{D_{\epsilon}\left(l^{(0)}\right)} \frac{1}{\left(l^{2}+M^{2}\right)^{2}}+\int_{D_{\epsilon}\left(l^{(1)}\right)} \frac{-1}{\left(l^{2}+M^{2}\right)^{2}}\right. \\
& \left.\quad+\int_{D_{r}} \Delta^{2}(l)\left(\cos l_{\mu} \cos l_{\nu}+\sin l_{\mu} \sin l_{\nu}\right)\right] \\
= & 0 .
\end{aligned}
$$

In this case, we thus find a species doubler.

4 We use quotation marks since it is not necessary for the propagator to behave as $p^{2}+M^{2}$ around the redundant pole(s). 
The situation is the same as in the hermitian case, (2.37), where $F_{\mu}(p)$ is given by (2.38) then

$$
\begin{aligned}
\mathcal{A}_{\mu}^{(1)}(p)= & -4 M^{2} \sum_{\alpha, \beta, \nu} \epsilon_{\alpha \beta} p_{\nu} \int_{l} \Delta^{2}(l)\left\{\delta_{\alpha \mu}\left(\cos l_{\mu}+\sin l_{\mu}\right)-\frac{2}{d} \sin l_{\mu}\right\} \\
& \times\left\{\delta_{\beta \nu}\left(\cos l_{\nu}+\sin l_{\nu}\right)-\frac{2}{d} \sin l_{\nu}\right\} \\
= & -4 M^{2} \sum_{\nu} \epsilon_{\mu \nu} p_{\nu} \int_{D_{\epsilon}\left(l^{(0)}\right)} \frac{1}{\left(l^{2}+M^{2}\right)^{2}} \\
& -4 M^{2} \sum_{\alpha, \beta, \nu} \epsilon_{\alpha \beta} p_{\nu} \int_{D_{\epsilon}\left(l^{(1)}\right)} \frac{\delta_{\alpha \mu} \delta_{\beta \nu}-\delta_{\alpha \mu}-\delta_{\beta \nu}+1}{\left(l^{2}+M^{2}\right)^{2}} \\
= & 0 .
\end{aligned}
$$

Here the pole at $(\pi / 2, \pi / 2)$ cancels the contribution from $p^{(0)}$, as it should be. This has also a species doubler. But the number of species doublers is reduced compare to the naive Dirac case which has $2^{2}=4$ poles.

\section{Discussion}

The discussion in the previous sections shows that any chirally invariant model must contain species doubler(s) provided the theory is well-regurarized in view of the condition (b). Although our conclusion has been checked in a two-dimensional model, it is straightforward to extend our scenario to four or higher dimensions.

As far as the number of species doubler(s) is concerned, the nonhermitian (in $d=2$ ) and ABCR models(in $d=2,4$ and 6 [14]) are most economical, since they have only one doubler. However if the condition (d), Direction-Interchange-Symmetry(DIS), is dropped, there open many possibilities to have less doubler [17] [18]. But if we do expect a better recovery of the Lorentz covariance together with an aethetic point of view, we do not adopt the model which breaks the DIS.

There might be many options which has the chiral symmetry, but in order to study whether the model is workable or not, it should be carefully checked that the model is well-regularized (the condition (b) ) together with the condition (c); otherwise we may encounter the computational trouble.

In a chirally invariant model, if we give up the gauge invariance on the lattice, we have the anomaly.(See [14] for example.) However we do think that on the lattice the gauge invariance should be kept all the time; otherwise we do lose the guiding principle for building up the lattice model. 


\section{Acknowledgements}

The authors are grateful to Jan Smit for informing us an earlier reference of Karsten, Wolfgang Bock and Maarten Golterman for fruitful suggestions. T. K. also thanks Don Petcher, Sergei Zenkin and Istvan Montvay for discussions.

\section{Appendix A. On Reflection Positivity}

In this appendix, we summarize the definition and consequences of reflection positivity and derive restrictions on the general class of free fermion actions (2.1).

We call the $d$-th direction of the euclidean spacetime the 'time' direction and suppose the time coordinate takes half-odd integer. Let $\mathcal{A}^{+}$be the set of funcitons at positive times which take their values in the Grassmann algebra generated by $\left\{\psi(n), \bar{\psi}(n) \mid n_{d}>0\right\}$. The set $\mathcal{A}^{-}$is similarly defined with $n_{d}<0$. Introduce an antilinear map $\Theta: \mathcal{A}^{+} \rightarrow \mathcal{A}^{-}$ defined as 19

$$
\begin{aligned}
& \Theta f(n)=[f(\vartheta n)]^{*}, \\
& \Theta \psi(n)=\bar{\psi}(\vartheta n) \gamma_{d}, \\
& \Theta \bar{\psi}(n)=\gamma_{d} \psi(\vartheta n), \\
& \Theta[A B]=(\Theta B)(\Theta A),
\end{aligned}
$$

where $\vartheta n \equiv\left(n_{1}, n_{2}, \ldots,-n_{d}\right)$ and the asterisk denotes the complex conjugate. Especially for a fermion bilinear form,

$$
\Theta\left[f(l) \bar{\psi}_{\alpha}(m) \chi_{\beta}(n)\right]=f(\vartheta l)^{*}\left(\bar{\chi}(\vartheta n) \gamma_{d}\right)_{\beta}\left(\gamma_{d} \psi(\vartheta m)\right)_{\alpha}
$$

where $\alpha$ and $\beta$ stand for the spinor and flavor indices.

Next we define a set $\mathcal{P}$ as the convex cone generated by $\left\{(\Theta A) A \mid A \in \mathcal{A}^{+}\right\}$. If the action $I$ satisfies $\exp (I) \in \mathcal{P}$, the physical Hilbert space with positive-definite metric can be induced by use of the functional integral on the lattice [19]. Here we call an action, $I$, to be refletion positive, when $\exp (I) \in \mathcal{P}$. We denote the entire cubic lattice as $\Lambda$ and divide it into the positive-time lattice, $\Lambda_{+}$, and the negative-time one, $\Lambda_{-}$. Any action which satisfies locality can always be decomposed as

$$
-I=I^{(+)}+I^{(-)}+\Delta I
$$

\footnotetext{
5 Although we consider free fermions, the inclusion of the link variables is straightforward.
} 
where $I^{( \pm)}$belongs to $\mathcal{A}^{ \pm}$and $\Delta I$ is a sum of products of the fields on $\Lambda_{+}$and those on $\Lambda_{-}$. Note that $\mathcal{P}$ is a multiplicative cone, i.e., if $A$ and $B$ are in $\mathcal{P}$, then $A B \in \mathcal{P}$. Hence, sufficient condition for $I$ to be reflection positive is

(a) $I^{(-)}=\Theta I^{(+)}$,

(b) $\Delta I \in \mathcal{P}$,

since $(\mathrm{a})$ means $\mathrm{e}^{I^{(+)}+I^{(-)}}=\left(\Theta \mathrm{e}^{I^{(+)}}\right) \mathrm{e}^{I^{(+)}} \in \mathcal{P}$ and $(\mathrm{b})$ guarantees the rest; $\mathrm{e}^{\Delta I} \in \mathcal{P}$.

Now we derive restrictions on the general class of fermion actions (2.1) from the reflection positivity. The mass term, $\sum_{n} \bar{\psi}(n) M \psi(n)$, is in $\mathcal{P}$ as far as $M$ is hermitian, so that we put $M=0$ for simplicity. Any action in this class can be decomposed into two terms, one of which contains timelike differences and the rest;

$$
-I=I_{t}+I_{s}
$$

and further each part can be divided into pieces like (A.3);

$$
\begin{aligned}
& I_{t}=I_{t}^{(+)}+I_{t}^{(-)}+\Delta I, \\
& I_{s}=I_{s}^{(+)}+I_{s}^{(-)} .
\end{aligned}
$$

where

$$
\begin{aligned}
& I_{t}^{(+)}=\sum_{k \geq 1} \sum_{\left\{n_{d} \geq 1 / 2\right\}}\left[\bar{\psi}(n) \Gamma_{d}^{(+; k)} \psi(n+k \cdot d)+\bar{\psi}(n+k \cdot d) \Gamma_{d}^{(-; k)} \psi(n)\right], \\
& I_{t}^{(-)}=\sum_{k \geq 1} \sum_{\left\{n_{d} \leq-k-1 / 2\right\}}\left[\bar{\psi}(n) \Gamma_{d}^{(+; k)} \psi(n+k \cdot d)+\bar{\psi}(n+k \cdot d) \Gamma_{d}^{(-; k)} \psi(n)\right], \\
& \Delta I= \sum_{k \geq 1} \sum_{\left\{-k+1 / 2 \leq n_{d} \leq-1 / 2\right\}}\left[\bar{\psi}(n) \Gamma_{d}^{(+; k)} \psi(n+k \cdot d)\right. \\
&\left.+\bar{\psi}(n+k \cdot d) \Gamma_{d}^{(-; k)} \psi(n)\right]
\end{aligned}
$$

and

$$
\begin{aligned}
I_{s}^{(+)}=\sum_{n_{d} \geq 1 / 2} & {\left[\sum_{i=1}^{d-1} \sum_{k \geq 1}\left[\bar{\psi}(n) \Gamma_{i}^{(+; k)} \psi(n+k \cdot i)+\bar{\psi}(n+k \cdot i) \Gamma_{i}^{(-; k)} \psi(n)\right]\right.} \\
& \left.+\bar{\psi}(n) \Gamma^{(0)} \psi(n)\right], \\
I_{s}^{(-)}=\sum_{n_{d} \leq-1 / 2} & {\left[\sum_{i=1}^{d-1} \sum_{k \geq 1}\left[\bar{\psi}(n) \Gamma_{i}^{(+; k)} \psi(n+k \cdot i)+\bar{\psi}(n+k \cdot i) \Gamma_{i}^{(-; k)} \psi(n)\right]\right.} \\
& \left.+\bar{\psi}(n) \Gamma^{(0)} \psi(n)\right]
\end{aligned}
$$


For $I$ to be reflection positive, the following conditions must be fulfilled:

(i) $I_{s}^{(-)}=\Theta I_{s}^{(+)}$,

(ii) $I_{t}^{(-)}=\Theta I_{t}^{(+)}$,

(iii) $\Delta I$ is expressed as $\Delta I=\sum_{j}\left(\Theta A_{j}\right) A_{j}$ with some $A_{j} \in \mathcal{A}^{+}$.

According to the definition (A.1), the condition (i) is satisfied iff

$$
\gamma_{d} \Gamma_{i}^{( \pm ; k)^{\dagger}} \gamma_{d}=\Gamma_{i}^{(\mp ; k)}(i=1, \ldots, d-1 ; k \geq 1) ; \quad \gamma_{d} \Gamma^{(0)^{\dagger}} \gamma_{d}=\Gamma^{(0)},
$$

and the condition (ii) is iff

$$
\gamma_{d} \Gamma_{d}^{( \pm ; k)^{\dagger}} \gamma_{d}=\Gamma_{d}^{( \pm ; k)} . \quad(k \geq 1)
$$

The consequence of the condition (iii) applied to the general class of actions is that any difference should be less than one. This can be shown as follows: each term in the righthand side of $\Delta I$ in (A.6) is composed of $\psi$ on $\Lambda_{+}$(or $\Lambda_{-}$) and $\bar{\psi}$ on $\Lambda_{-}\left(\right.$or $\Lambda_{+}$). Except for the $k=1$ term, any term is 'asymmetric' with respect to the reflection, i.e., cannot be written in the form $(\Theta A) A$ with some $A$ on either $\Lambda_{+}$or $\Lambda_{-}$. There might remain a possibility that sum of the terms, not one of them, could be written as $\sum_{j}\left(\Theta A_{j}\right) A_{j}$ where $A_{j}$ is a linear combination of $\psi$ on $\Lambda_{+}$or that of $\bar{\psi}$ on $\Lambda_{+}$.

Let $K$ be the maximum integer such that any $\Gamma_{d}^{( \pm ; k)}$ with $k>K$ vanishes. If $\Delta I$ meets the condition (iii), it is generally expressed by introducing a set of elements in $\mathcal{A}^{+}$;

$$
\begin{aligned}
A_{i} & =\sum_{n_{d}=1 / 2}^{K-1 / 2} \alpha_{i}\left(n_{d}\right) \psi\left(n_{d}\right), \\
B_{j} & =\sum_{n_{d}=1 / 2}^{K-1 / 2} \bar{\psi}\left(n_{d}\right) \beta_{j}^{\dagger}\left(n_{d}\right),
\end{aligned}
$$

as

$$
\Delta I=\sum_{i=1}^{I}\left(\Theta A_{i}\right) A_{i}+\sum_{j=1}^{J}\left(\Theta B_{j}\right) B_{j},
$$

where $I$ and $J$ are some finite numbers and $\alpha_{i}$ and $\beta_{j}$ are complex matrices in spinor (and flavor) space. Here we have suppressed the spatial coordinates. From (A.11), we have

$$
\begin{aligned}
& \sum_{i} \gamma_{d} \alpha_{i}^{\dagger}\left(-n_{d}\right) \alpha_{i}\left(n_{d}+k\right)=\Gamma_{d}^{(+; k)}, \\
& \text { for }-k+1 / 2 \leq n_{d} \leq-1 / 2 \quad \text { with } 1 \leq k \leq K, \\
& \sum_{i} \gamma_{d} \alpha_{i}^{\dagger}\left(-n_{d}\right) \alpha_{i}\left(n_{d}+k+K\right)=0, \\
& \text { for }-K+1 / 2 \leq n_{d} \leq-k-1 / 2 \quad \text { with } 1 \leq k \leq K-1(K \geq 2),
\end{aligned}
$$


and similar equations for $\beta_{j}$ and $\Gamma_{d}^{(-; k)}$. The second equation requires all $\alpha_{i}$ but $\alpha_{i}(1 / 2)$ vanish. Hence the difference in the time direction should be less than one; $\Gamma_{d}^{( \pm ; k)}=0 ; k \geq 2$. Further the remaining $\Gamma_{d}^{( \pm ; 1)}$ must be written as

$$
\begin{aligned}
& \sum_{i} \alpha_{i}^{\dagger} \alpha_{i}=\gamma_{d} \Gamma_{d}^{(+; 1)}, \\
& \sum_{j} \beta_{j}^{\dagger} \beta_{j}=-\Gamma_{d}^{(-; 1)} \gamma_{d},
\end{aligned}
$$

with some complex matrices $\alpha_{i}$ and $\beta_{j}$. This condition, applied to the Wilson fermion action, requires that $\left(1 \pm r \gamma_{d}\right) / 2$ must be positive semi-definite. It immediately means that $|r| \leq 1$, since the eigenvalues of $\left(1 \pm r \gamma_{d}\right) / 2$ are either $(1+r) / 2$ or $(1-r) / 2$. 


\section{References}

[1] H. B. Nielsen and M. Ninomiya, Nucl. Phys. B185, 20 (1981); B193, 173 (1981); (E) B193 , 541 (1981).

[2] M. F. L. Golterman, Nuc. Phys. B (Proc. Suppl.) 20, 528 (1991); I. Montvay, Plenary Talk at Lattice '91(to be published in Nucl. Phys. B (Proc. Suppl.))

[3] K. G. Wilson, in New Phenomena in Subnuclear Physics, e.d. A. Zichichi, Erice Lecture 1975, (Plenum 1977).

[4] C. Pryor, Phys. Rev. D43, 2669 (1991).

[5] P. D. V. Swift, Phys. Lett. B145, 256 (1984); J. Smit, Acta Physica Polonica B17, 531 (1986).

[6] K. Funakubo and T. Kashiwa, Phys. Rev. Lett,60, 2113 (1988).

[7] S. Aoki, Phys. Rev. Lett. 60, 2109 (1988).

[8] M. F. L. Golterman and D. N. Petcher, Phys. Lett. B225 159 (1989)

[9] T. W. Chiu, Phys. Lett. B217, 151 (1989); B206, 510 (1988); S. J. Perantonius and J. F. Wheater, Nucl. Phys. B295, [FS 21], 443 (1988).

[10] I. Montvay, Phys. Lett. B199, 89 (1987).

[11] M. Gross, G. P. Lepage and P. E. L. Rakow, Phys. Lett. B197, 183 (1987).

[12] K. Funakubo and T. Kashiwa, to be published in Nucl. Phys. B (Lattice '91 Proc. Suppl.)

[13] L. H. Karsten and J. Smit, Nucl. Phys. B183, 103 (1981); T. Kashiwa and H. So, Prog. Theor. Phys. 73, 762 (1985).

[14] J. -L. Alonso, P. Boucaud, J. -L. Cortes and E. Rivas, Phys. Lett. B201, 340 (1988); Phys. Rev. D40, 4123 (1989).

[15] Y. Ohnuki and T. Kashiwa, Prog. Theor. Phys. 60, 548 (1978); T. Kashiwa and H. So, Prog. Theor. Phys. 73, 762 (1985)

[16] T. Kashiwa and H. So, Prog. Theor. Phys. 73, 762 (1985); K. Funakubo and T. Kashiwa, Phys. Rev. D38, 2602 (1988).

[17] L. H. Karsten, Phys. Lett. 104B, 315 (1981)

[18] F. Wilczek, Phys. Rev. Lett. 59, 2397 (1987)

[19] K. Osterwalder and E. Seiler, Ann. Phys. 110 (1978) 440. 